\title{
A spectral sequence for Khovanov homology with an application to $(3, q)$-torus links
}

\author{
PAUL TURNER
}

\begin{abstract}
We extend the skein exact sequence of Khovanov homology to a spectral sequence which converges to Khovanov homology. We apply this to calculate the rational Khovanov homology of three-stranded torus links.
\end{abstract}

57M25; 57M27, 55T99

\section{Introduction}

When compared to the Jones polynomial, Khovanov's homology for links shows advantages similar to those enjoyed by ordinary homology (of spaces) over the Euler characteristic: it is a stronger invariant, it is richer (eg the integral theory reveals torsion) and perhaps most importantly it has nice functorial properties. Also, like ordinary homology, calculations made directly from the definitions quickly become unmanageable. That said, there are some ingenious algorithms and methods which now allow computer calculations in good cases for diagrams with 60 or so crossings (see BarNatan [3]). Unlike ordinary homology, there is a dearth of theoretical computational tools for Khovanov homology. One would dearly love the battery of long exact sequences and spectral sequences available to the algebraic topologist. Thus, currently it is still rather difficult to compute Khovanov homology for any infinite class of links.

There is, none the less, one long exact sequence used in Khovanov homology, the skein exact sequence, which is implicit in Khovanov [4] and explicit in Viro [9]. Recall that a given crossing of a link diagram may be resolved in one of two possible ways: to the 0 -smoothing or to the 1 -smoothing as indicated below.
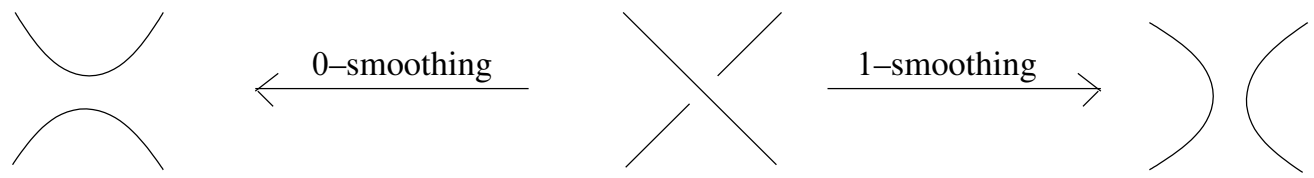

Considering all crossings of a diagram, the Khovanov complex is formed by applying a certain $1+1$-dimensional topological quantum field theory to the resulting $2^{n}$ possible 
ways of smoothing the $n$ crossings of the diagram. The Khovanov homology (a bigraded module) is obtained as the homology of this complex. Choosing a particular crossing, the underlying module of the Khovanov complex $C(D)$ can be decomposed as

$$
C(D) \cong C\left(D^{\prime}\right) \oplus C\left(D^{\prime \prime}\right)
$$

where $D^{\prime}$ and $D^{\prime \prime}$ are the diagrams obtained from $D$ by resolving the chosen crossing into a 0 - and 1 -smoothing respectively. In fact more is true and there is a short exact sequence of Khovanov complexes

$$
0 \rightarrow C\left(D^{\prime \prime}\right) \rightarrow C(D) \rightarrow C\left(D^{\prime}\right) \rightarrow 0
$$

which gives rise to a long exact sequence in homology (with suitable gradings).

There is also a useful spectral sequence which converges to Lee's variant of Khovanov homology and has rational Khovanov homology appearing at the $E_{2}-$ page; see Lee [5] and Rasmussen [6]. Since Lee's homology is easy to compute [5] (depending only on the linking matrix of the link) one can, by reverse engineering, obtain some information about Khovanov homology itself. For example one can prove Bar-Natan's conjecture (about the form of rational Khovanov homology) for alternating links [5].

One can repeatedly apply the skein long exact sequence, but it requires careful book keeping and considerable redundancy. The spectral sequence developed in this paper streamlines the iterated application of this long exact sequence. We start with a collection of $m$ ordered crossings of an $n$-crossing diagram $D$. For $1 \leq k \leq m$ let $D_{k}$ be the diagram obtained from $D$ by resolving the crossings $1, \ldots, k$ to 1 -smoothings and let $\widetilde{D}_{k}$ be the diagram obtained from $D$ by resolving the crossings $1, \ldots, k-1$ to 1 -smoothings and crossing $k$ to a 0 -smoothing. The idea, or hope, is that the diagrams $\widetilde{D}_{k}$ and $D_{m}$ might be simpler to handle than the diagram $D$ and that we can compute $K H^{*, *}(D)$ in terms of the $K H^{*, *}\left(\widetilde{D}_{k}\right)$ and $K H^{*, *}\left(D_{m}\right)$. This is achieved by the spectral sequence of the title where there is one spectral sequence for each $q$-grading of Khovanov homology. By defining appropriate constants $a_{k}, b_{k}, \widetilde{a}_{k}, \widetilde{b}_{k}, A_{k}$, and $B_{k}$ we arrive at the following result where $j$ is fixed.

Proposition 2.2 There is a spectral sequence $\left(E_{r}^{*, *}, d_{r}: E_{r}^{s, t} \rightarrow E_{r}^{s+r, t-r+1}\right)$ converging to $K H^{*, j}(D)$ with $E_{1}$-page given by

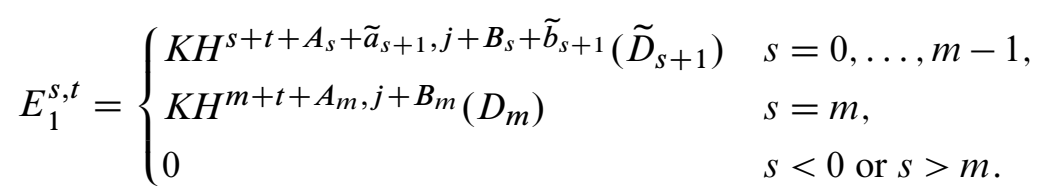

As a sample application of this spectral sequence we compute, in Theorem 3.1, the rational Khovanov homology of $(3, q)$-torus links. It is easy to guess what the result is, 
based on available computer calculations, but by combining the new spectral sequence with Lee's spectral sequence we prove the result for all $q$.

\section{The spectral sequence}

Let $R$ be a commutative ring with unit and let $D$ be an oriented link diagram with $n$ crossings. As is now familiar one can construct the Khovanov complex by placing the $2^{n}$ smoothings of $D$ on the vertices of the cube $\{0,1\}^{n}$. To each smoothing $\alpha$ one then assigns the $R$-module $V_{\alpha}=V^{\otimes k_{\alpha}}\left\{r_{\alpha}\right\}$ where $k_{\alpha}$ is the number of circles in the smoothing, $r_{\alpha}$ is the number of 1's in $\alpha$ and shifts are defined by $(W\{l\})^{m}=W^{m-l}$. The module $V$ is the graded, rank two, free $R$-module with generators 1 and $x$ in degree 1 and -1 respectively. The underlying module of the unnormalised Khovanov complex $\bar{C}^{*, *}(D)$ is defined by

$$
\bar{C}^{i, *}(D)=\bigoplus_{\substack{\alpha \in\{0,1\}^{n} \\ r_{\alpha}=i}} V_{\alpha} .
$$

The construction of the differential is by now well known (see, for example, Khovanov [4] or Bar-Natan [1]) and uses a Frobenius algebra structure on $V$.

Bigraded complexes may be shifted in each of the degrees and for a bigraded module $W^{* *}$ we define

$$
\left(W^{*, *}[l]\{m\}\right)^{i, j}=W^{i-l, j-m} .
$$

Suppose $D$ has $n^{+}$positive crossings and $n^{-}$negative crossings, then the normalised Khovanov complex $C^{*, *}(D)$ is defined by

$$
C^{i, j}(D)=\left(\bar{C}^{*, *}(D)\left[-n^{-}\right]\left\{n^{+}-2 n^{-}\right\}\right)^{i, j}
$$

and the Khovanov homology of $D$ is the homology of this complex.

Now let us consider a collection of $m$ crossings of the diagram $D$ and number these $1, \ldots, m$. For $k=1, \ldots, m$ let $D_{k}$ be the diagram obtained from $D$ by resolving the crossings $1, \ldots, k$ to 1 -smoothings and let $\widetilde{D}_{k}$ be the diagram obtained from $D$ by resolving the crossings $1, \ldots, k-1$ to 1 -smoothings and crossing $k$ to a 0 -smoothing. We also define $D_{0}$ and $\widetilde{D}_{0}$ to be the original diagram $D$.

There is a decomposition of modules

$$
\bar{C}^{i, j}\left(D_{k-1}\right)=\bar{C}^{i, j}\left(\widetilde{D}_{k}\right) \oplus \bar{C}^{i-1, j-1}\left(D_{k}\right)
$$


and in fact $\bar{C}^{*-1, *-1}\left(D_{k}\right)$ is a subcomplex of $\bar{C}^{*, *}\left(D_{k-1}\right)$. Thus, there is a short exact sequence

$$
0 \longrightarrow \bar{C}^{*, *}\left(D_{k}\right)[1]\{1\} \longrightarrow \bar{C}^{*, *}\left(D_{k-1}\right) \longrightarrow \bar{C}^{*, *}\left(\widetilde{D}_{k}\right) \longrightarrow 0 .
$$

This is just the usual short exact sequence giving the skein long exact sequence mentioned in the introduction applied to the diagram $D_{k}$ by resolving the $k$-th crossing in our set of $m$ crossings.

We now discuss orientations for the diagrams $D_{k}$ and $\widetilde{D}_{k}$. Suppose that we already have an orientation for $D_{k-1}$. If the $k$-th crossing is positive then $\widetilde{D}_{k}$ inherits an orientation because for positive crossings the 0 -smoothing is the oriented resolution. There is no orientation of $D_{k}$ consistent with the orientation of $D_{k-1}$ so choose any orientation for $D_{k}$. Similarly if the $k$-th crossing is negative then $D_{k}$ inherits an orientation and we choose any orientation for $\widetilde{D}_{k}$. The diagram $D=D_{0}=\widetilde{D}_{0}$ comes with an orientation so the process above has somewhere to start.

Now for $k=0, \ldots, m$ define

$$
\begin{aligned}
& n_{k}^{+}=\text {number of positive crossings in } D_{k}, \\
& n_{k}^{-}=\text {number of negative crossings in } D_{k}, \\
& \tilde{n}_{k}^{+}=\text {number of positive crossings in } \widetilde{D}_{k}, \\
& \tilde{n}_{k}^{-}=\text {number of negative crossings in } \widetilde{D}_{k} .
\end{aligned}
$$

We define additional constants associated to $D_{k}$ and $\widetilde{D}_{k}$ as follows.

If the $k$-th crossing is positive (as a crossing in the oriented diagram $D_{k-1}$ ) then set

$$
a_{k}=n_{k-1}^{-}-n_{k}^{-}-1 \text { and } \tilde{a}_{k}=0 .
$$

If the $k$-th crossing is negative then set

$$
a_{k}=0 \quad \text { and } \quad \tilde{a}_{k}=n_{k-1}^{-}-\tilde{n}_{k}^{-} .
$$

For convenience we also define (for positive and negative crossings)

$$
b_{k}=3 a_{k}+1 \quad \text { and } \quad \tilde{b}_{k}=3 \tilde{a}_{k}-1 .
$$

These constants help us to write down the short exact sequence (1) in terms of normalised Khovanov homology.

Proposition 2.1 For each $k=1, \ldots, m$ there is a short exact sequence of complexes

$$
0 \longrightarrow C^{*, *}\left(D_{k}\right)\left[-a_{k}\right]\left\{-b_{k}\right\} \longrightarrow C^{*, *}\left(D_{k-1}\right) \longrightarrow C^{*, *}\left(\tilde{D}_{k}\right)\left[-\tilde{a}_{k}\right]\left\{-\tilde{b}_{k}\right\} \longrightarrow 0
$$


Proof We shift the entire sequence (1) by $\left[-n_{k-1}^{-}\right]\left\{n_{k-1}^{+}-2 n_{k-1}^{-}\right\}$. One can then readily verify (treating positive and negative crossings separately) that

$$
\begin{aligned}
-n_{k-1}^{-}+1 & =-n_{k}^{-}-a_{k}, \\
-n_{k-1}^{-} & =-\tilde{n}_{k}^{-}-\tilde{a}_{k}, \\
n_{k-1}^{+}-2 n_{k-1}^{-}+1 & =n_{k}^{+}-2 n_{k}^{-}-b_{k}, \\
n_{k-1}^{+}-2 n_{k-1}^{-} & =\tilde{n}_{k}^{+}-2 \tilde{n}_{k}^{-}-\widetilde{b}_{k}
\end{aligned}
$$

from which the result easily follows.

We now define

$$
A_{k}=\sum_{i=1}^{k} a_{i} \text { and } B_{k}=\sum_{i=1}^{k} b_{i}=3 A_{k}+k
$$

and set $A_{0}=B_{0}=0$.

From now on we fix $j$. We define a filtration on $C^{*, j}(D)$ by

$$
F^{k} C^{*, j}(D)=C^{*, j}\left(D_{k}\right)\left[-A_{k}\right]\left\{-B_{k}\right\} \quad k=0, \ldots, m .
$$

It follows immediately from Proposition 2.1 that $F^{k} C^{*, j}(D) \subset F^{k-1} C^{*, j}(D)$ and for $k>m$ we set $F^{k} C^{*, j}(D)=0$. It is clear that the filtration is bounded and so there is an associated spectral sequence.

Proposition 2.2 There is a spectral sequence $\left(E_{r}^{*, *}, d_{r}: E_{r}^{s, t} \rightarrow E_{r}^{s+r, t-r+1}\right)$ converging to $K H^{*, j}(D ; R)$ with $E_{1}$-page given by

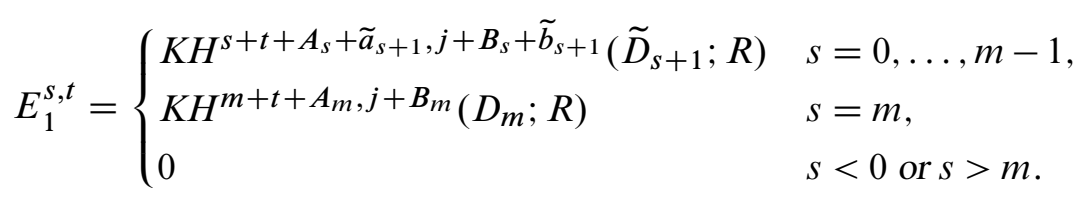

Proof Using the filtration above there is a spectral sequence with $E_{0}$-page given by

$$
E_{0}^{s, t}=\frac{F^{s} C^{s+t, j}(D)}{F^{s+1} C^{s+t, j}(D)} .
$$

To understand this quotient we can apply Proposition 2.1 to see that there is a short exact sequence for each $s$ in the range $0 \leq s<m$ given by

$$
\begin{aligned}
& 0 \longrightarrow C^{s+t+A_{s}, j+B_{s}}\left(D_{s+1}\right)\left[-a_{s+1}\right]\left\{-b_{s+1}\right\} \longrightarrow C^{s+t+A_{s}, j+B_{s}}\left(D_{s}\right) \\
& \longrightarrow C^{s+t+A_{s}, j+B_{s}}\left(\tilde{D}_{s+1}\right)\left[-\tilde{a}_{s+1}\right]\left\{-\tilde{b}_{s+1}\right\} \longrightarrow 0 .
\end{aligned}
$$


Writing this in terms of the filtration groups we have

$0 \longrightarrow F^{s+1} C^{s+t, j}(D) \longrightarrow F^{s} C^{s+t, j}(D)$

$$
\longrightarrow C^{s+t+A_{s}+\tilde{a}_{s+1}, j+B_{s}+\tilde{b}_{s+1}}\left(\widetilde{D}_{s+1}\right) \longrightarrow 0 .
$$

Since these give a short exact sequence of complexes the differential $d_{0}$, which is induced by the differential on $F^{s} C^{*, j}(D)$, can be identified with the differential on the right hand side, that is, in the complex $C^{*, *}\left(\widetilde{D}_{s+1}\right)$. In particular the homology of $E_{0}^{*, *}$ is given by the homology (in suitable gradings) of $C^{*, *}\left(\widetilde{D}_{s+1}\right)$, namely the Khovanov homology of $\widetilde{D}_{s+1}$.

When $s=m$ we have $E_{0}^{m, t}=F^{m} C^{m+t, j}(D)=C^{m+t+A_{m}, j+B_{m}}\left(D_{m}\right)$ and so $E_{1}$ is again as claimed.

The differential $d_{1}$ on the $E_{1}$-page can be understood as follows. There is a decomposition (of modules)

$$
\begin{array}{r}
C^{*, *}\left(D_{s}\right)=C^{*+\tilde{a}_{s+1}, *+\tilde{b}_{s+1}}\left(\widetilde{D}_{s+1}\right) \\
\oplus C^{*+a_{s+1}+\tilde{a}_{s+2}, *+b_{s+1}+\widetilde{b}_{s+2}\left(\widetilde{D}_{s+2}\right)} \\
\oplus C^{*+a_{s+1}+a_{s+2}, *+b_{s+1}+b_{s+2}}\left(D_{s+2}\right)
\end{array}
$$

and with respect to this the differential on $C^{*, *}\left(D_{S}\right)$ can be written as a matrix

$$
\left(\begin{array}{ccc}
\tilde{\delta}_{s+1} & 0 & 0 \\
\delta & \tilde{\delta}_{s+2} & 0 \\
\delta^{\prime} & \delta^{\prime \prime} & \delta_{s+2}
\end{array}\right) .
$$

The differential on the $E_{1}$-page of the spectral sequence is the map

$$
\delta: C^{*+\tilde{a}_{s+1}, *+\widetilde{b}_{s+1}}\left(\widetilde{D}_{s+1}\right) \rightarrow C^{*+a_{s+1}+\tilde{a}_{s+2}, *+b_{s+1}+\widetilde{b}_{s+2}}\left(\widetilde{D}_{s+2}\right)
$$

in the above matrix.

Note that if $m=1$ then the $E_{1}$-page is concentrated in columns $s=0$ and $s=1$ and so collapses at the $E_{2}$-page for dimensional reasons. The differential on the $E_{1}$-page is precisely the boundary map in the usual long exact sequence. Indeed one can always assemble such a situation into a long exact sequence.

It is worth commenting that the essential ingredient for the construction of the spectral sequence is the cube construction of link homology, not the particular variant of link homology we choose to consider. Thus, for example, one may set up similar spectral sequences for Khovanov-Rozansky homology. 
Computing the differentials in the above spectral sequence is tantamount to knowing the whole Khovanov complex so it is desirable to have some other way of proceeding. Working over $\mathbb{Q}$ there is a technique which appears to be remarkably successful which involves Lee's spectral sequence. Recall that Lee theory [5] is a variant of rational Khovanov homology obtained from the same underlying vector spaces but using a different differential (based on a different Frobenius algebra). Lee theory is a singly graded (filtered) theory and we denote it by Lee* $(L)$. We summarise the results we need about Lee theory in the following proposition.

Proposition 2.3 Let $L$ be an oriented link with $k$ components $L_{1}, L_{2}, \ldots, L_{k}$.

(1) The dimension of $\operatorname{Lee}^{*}(L)$ is $2^{k}$.

(2) For every orientation $\theta$ of $L$ there is a generator of homology in degree

$$
2 \times \sum_{l \in E, m \in \bar{E}} 1 \mathrm{k}\left(L_{l}, L_{m}\right)
$$

where $E \subset\{1,2, \cdots, k\}$ indexes the set of components of $L$ whose original orientation needs to be reversed to get the orientation $\theta$ and $\bar{E}=\{1, \ldots, k\} \backslash E$. The linking numbers $\operatorname{lk}\left(L_{l}, L_{m}\right)$ are the linking number (for the original orientation) between component $L_{l}$ and $L_{m}$.

(3) There is a spectral sequence $\left(E_{r}^{*, *}, d_{r}: E_{r}^{s, t} \rightarrow E_{r}^{s+r, t-r+1}\right)$ converging to Lee $^{*}(L)$ with $E_{2}$-page given by the rational Khovanov homology of $L$.

The spectral sequence in (iii) has differential $d_{i}=0$ for $i$ odd. If we index the $E_{2 i}-$ page by the usual indexing of the Khovanov homology (rather than of the spectral sequence) the differential $d_{2 i}$ is of bidegree $(1,4 i)$.

To bypass computing the differentials in the spectral sequence of Proposition 2.2 we can use the $E_{1}$-page for $q$-grading $j$ to produce an approximation to $K H^{*, j}(D)$ with some definite generators (those for which it is already clear from the $E_{1}$-page that they survive to $E_{\infty}$ ) and also some additional possible generators. It is not sure that the possible generators are in fact generators because there may be higher differentials in the spectral sequence killing them. We can then assemble these over $j$ to form our approximation to $K H^{*, *}(D)$ and use Lee's spectral sequence, in particular the knowledge of the simple $E_{\infty}$-page, to determine whether or not the possible generators are killed or not. By playing off one spectral sequence against another in this way, we do not actually have to explicitly compute any differentials in either spectral sequence.

Reduced Khovanov homology $\mathrm{KH}_{r}^{*, *}{ }_{(-)}$is also produced via a cube and so there is a spectral sequence analogous to the above. Indeed all the indices and constants are 
identical to the above and so one can just rewrite Proposition 2.2 replacing $K H^{*, *}(-)$ with $K H_{r}^{*, *}(-)$. Unfortunately there is no reduced Lee theory so the method above to avoid explicit computation of differentials will fail. On the other hand there is another deformation of Khovanov homology due to Bar-Natan [2] defined over $\mathbb{Z}, \mathbb{Q}$ or any $\mathbb{F}_{p}$ ( $p$ a prime) satisfying all the properties of Proposition 2.3. These theories do have a reduced version and there is a spectral sequence whose $E_{1}$-page is given by reduced Khovanov homology, converging to reduced Bar-Natan theory. By indexing as for Khovanov homology the differential $d_{i}$ on the $E_{i}$-page is of bidegree $(1,2)$. Moreover $d_{1}$ can be understood explicitly as explained in [8]. Thus, we can again attempt to bypass explicit computation of differentials in the (reduced version of the) spectral sequence of Proposition 2.2 by the same method as above, but now using reduced Bar-Natan theory. In practice this situation is considerably less restrictive than using Lee theory, stemming from the differentials having bidegree $(1,2)$ rather than $(1,4)$. However, it is worth noting, that in principle this does give a way of tackling the reduced integral Khovanov homology.

\section{The rational Khovanov homology of $(3, q)$-torus links}

In this section we work over $\mathbb{Q}$ and write $K H^{*, *}(D)$ for $K H^{*, *}(D ; \mathbb{Q})$. Our interest is with the torus links $T(3, q)$ which we take to have negative crossings. We consider the diagram for $T(3, q)$ obtained as the closure of a three stranded braid as shown in Figure 2. When $q$ is a multiple of 3 then $T(3, q)$ is a three component link, otherwise $T(3, q)$ is a knot.

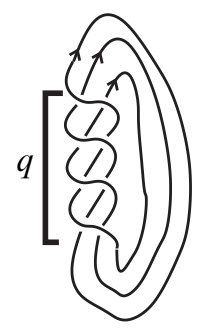

Figure 2: Diagram for torus link

We note that for a knot Lee theory has two generators in degree zero. For the $(3,3 N)$ torus link Lee theory has two generators in degree zero and six generators in degree $-4 N$.

We now apply the spectral sequence constructed in the previous section to compute $K H^{*, *}(T(3, q))$. As is customary we present the result by way of a table where 
$K H^{i, j}(-)$ occurs in the column labelled $i$ and row labelled $j$. The rows in these tables go up in steps of two. The vector space consisting of the direct sum of $k$ copies of $\mathbb{Q}$ is denoted in the table by $k$.

Theorem 3.1 Let $N$ be an integer, $N \geq 1$.

(1) The rational Khovanov homology of the $(3,3 N)$-torus link is given by:

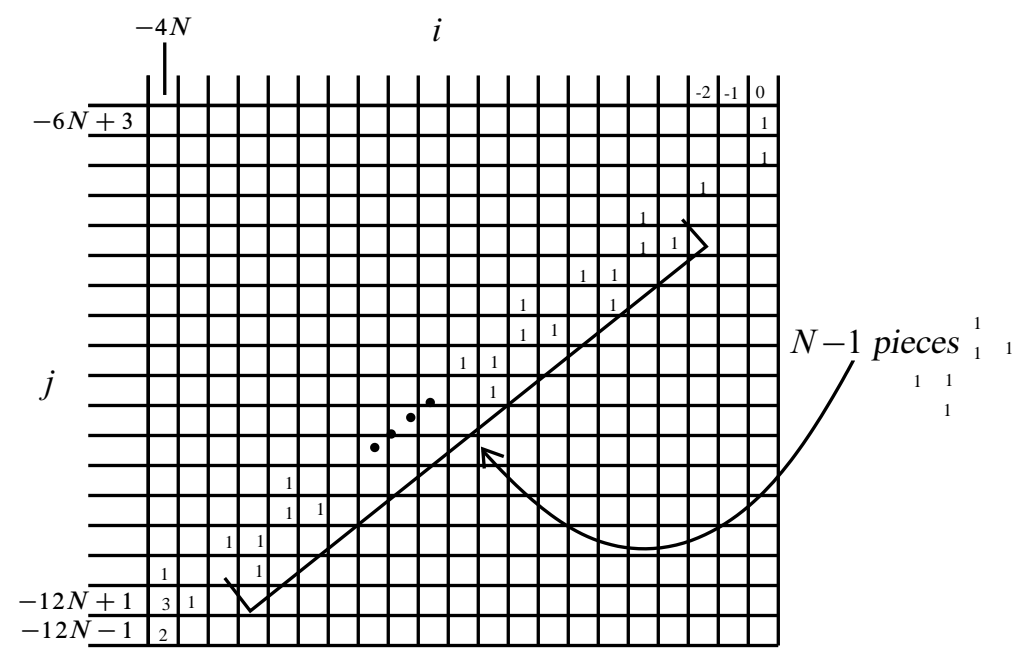

(2) The rational Khovanov homology of the $(3,3 N+1)$-torus knot is given by:

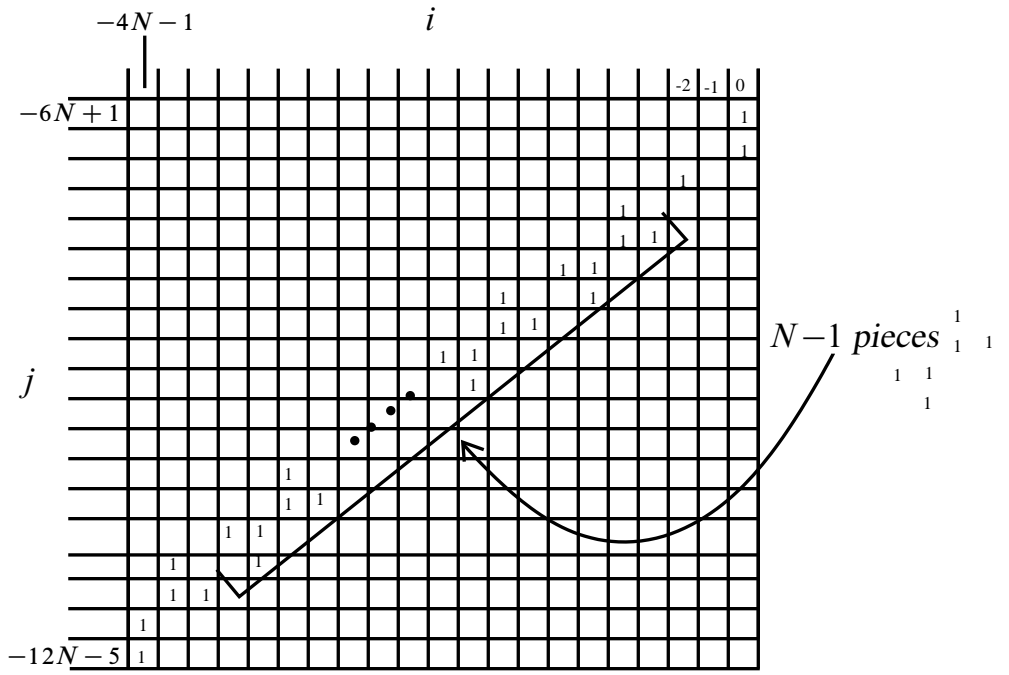


(3) The rational Khovanov homology of the (3, $3 N-1)$-torus knot is given by:

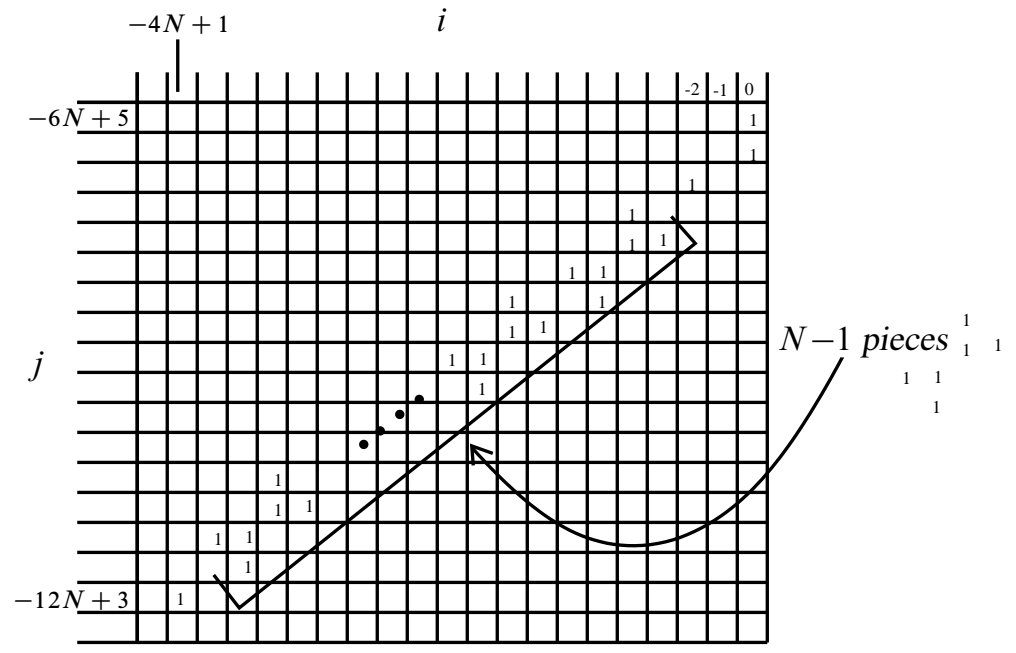

Proof The proof is by induction and consists of three claims:

Claim 1 If the result is true for $T(3,3 N-1)$ then the result is true for $T(3,3 N)$.

Claim 2 If the result is true for $T(3,3 N)$ then the result is true for $T(3,3 N+1)$.

Claim 3 If the result is true for $T(3,3 N+1)$ then the result is true for $T(3,3(N+$ 1) -1$)$.

Proof of Claim 1 We will calculate the Khovanov homology of the link $T(3,3 N)$ under the assumption that the Khovanov homology of $T(3,3 N-1)$ is as given in the statement of the theorem. Consider the set of crossings consisting of the two top crossings in the braid diagram (so $m=2$ ). We have diagrams as presented in Figure 6 . Note that $D_{2}=T(3,3 N-1)$ and it is easy to see that $\widetilde{D}_{1} \sim U \sqcup U$ and $\widetilde{D}_{2} \sim U$, where $U$ is the unknot. Using the orientations shown in Figure 6 one computes

$$
\tilde{n}_{1}^{+}=4 n-1, \quad \tilde{n}_{1}^{-}=2 N, \quad \tilde{a}_{1}=4 N, \quad \tilde{b}_{1}=12 N-1
$$

and

$$
\tilde{n}_{2}^{+}=4 n-1, \quad \tilde{n}_{2}^{-}=2 N-1, \quad \tilde{a}_{2}=4 N, \quad \tilde{b}_{2}=12 N-1 .
$$

From Proposition 2.2 we have for fixed $j$,

$$
\begin{aligned}
& E_{1}^{0, t}=K H^{t+4 N, j+12 N-1}(U \sqcup U), \\
& E_{1}^{1, t}=K H^{t+4 N+1, j+12 N}(U), \\
& E_{1}^{2, t}=K H^{t+2, j+2}(T(3,3 N-1)) .
\end{aligned}
$$




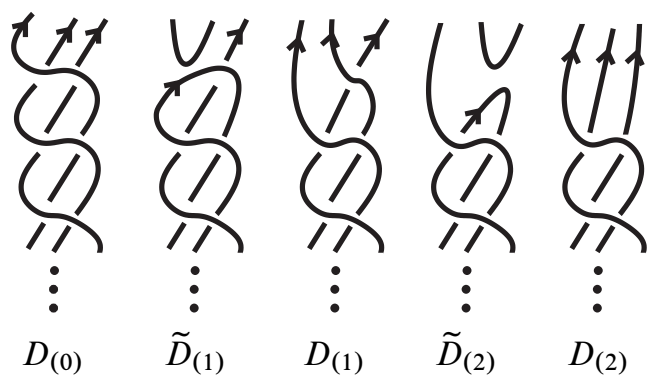

Figure 6: Diagrams for Claim 1

When $s=0$ we see that $E_{1}^{0, t}=0$ unless $t=-4 N$ and $j=-12 N-1, j=-12 N+1$ or $j=-12 N+3$. Similarly, $E_{1}^{1, t}=0$ unless $t=-4 N-1$ and $j=-12 N-1$ or $j=-12 N+1$ and $E_{1}^{2, t}$ is zero unless $-12 N+1 \leq j \leq-6 N+3$.

For $j>-12 N+3$ the $E_{1}$-term of the spectral sequence is concentrated in the column $s=2$ and hence collapses for dimensional reasons. Thus,

$$
K H^{i, j}(T(3,3 N)) \cong E_{1}^{2, i-2}=K H^{i, j+2}(T(3,3 N-1)) .
$$

We need to consider the three cases $j=-12 N-1, j=-12 N+1$ and $j=-12 N+3$. The $E_{1}$ pages are give in Figure 7.

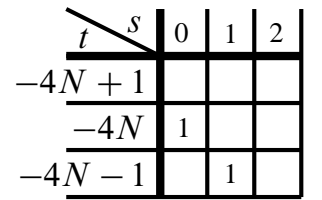

$j=-12 N-1$

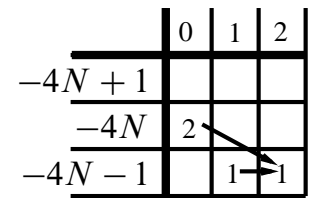

$j=-12 N+1$

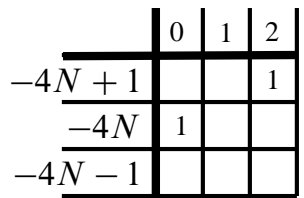

$j=-12 N+3$

Figure 7: $E_{1}$-pages for Claim 1

For $j=-12 N-1$ and $j=-12 N+3$ there are no differentials for dimensional reasons thus the spectral sequence collapses at $E_{1}$. For $j=-12 N+1$ there is a possible $d_{1}$ and a possible $d_{2}$ (but not both) as shown in Figure 7. Thus for $T(3,3 N)$ we have the situation presented in Figure 8, where possible generators are circled.

The three possible generators in bidegree $(-4 N,-12 N+1)$ must all indeed be generators because we require at least six generators in homological degree $-4 N$. This is because Lee theory in this degree has six generators and due to Lee's spectral sequence these must show up in Khovanov homology. 


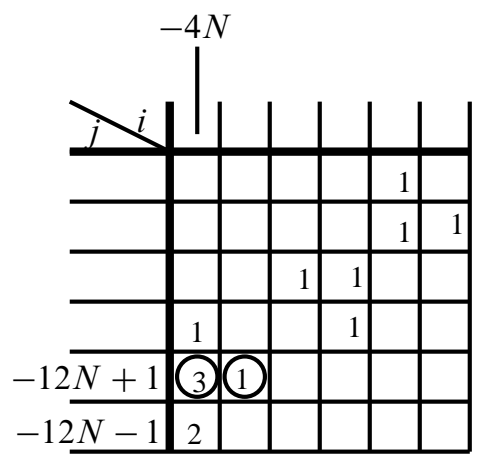

Figure 8: Generators and possible generators in Khovanov homology for $T(3,3 N)$

The possible generator in bidegree $(-4 N+1,-12 N+1)$ is also a generator. If we look at the $E_{1}$ page for $j=-12 N+1$ then since the three generators on the line $s+t=-4 N$ survive until $E_{\infty}$ (as shown in the previous paragraph) then there is nothing to kill the remaining generator. (Alternatively, the generator in bidegree $(-4 N+2,-12 N+5)$ must be killed in Lee's spectral sequence and the only possible way this can happen is for the possible generator in bidegree $(-4 N+1,-12 N+1)$ to be present. To see this, recall that indexed this way the differential $d_{2 i}$ in Lee's spectral sequence has bidegree $(1,4 i)$.)

Thus we end up computing $K H^{*, *}(T(3,3 N))$ as presented in the theorem.

Proof of Claim 2 Consider the link $T(3,3 N+1)$ and as above take the set of crossings to be the two top crossings in the braid diagram. We have diagrams as presented in Figure 9.

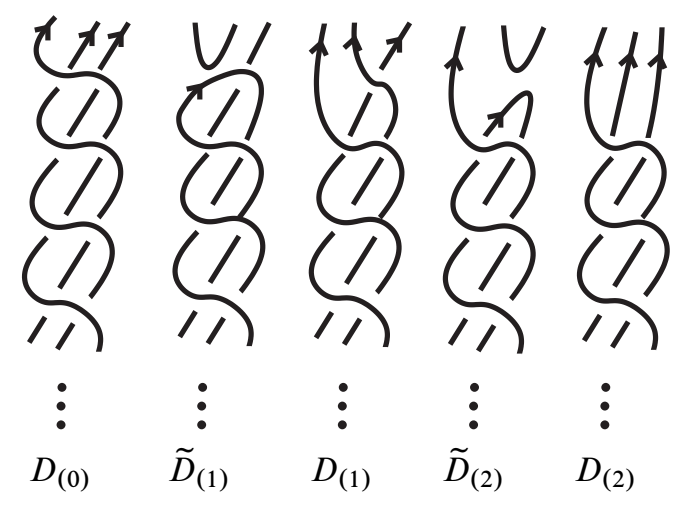

Figure 9: Diagrams for Claim 2 
Note that $D_{2}=T(3,3 N)$ and it is easy to show $\widetilde{D}_{1} \sim U$ and $\widetilde{D}_{2} \sim U \sqcup U$. Using the orientations shown in Figure 9 one computes

$$
\tilde{n}_{1}^{+}=4 N, \quad \tilde{n}_{1}^{-}=2 N+1, \quad \tilde{a}_{1}=4 N+1, \quad \tilde{b}_{1}=12 N+2
$$

and

$$
\tilde{n}_{2}^{+}=4 N, \quad \tilde{n}_{2}^{-}=2 N, \quad \tilde{a}_{2}=4 N+1, \quad \tilde{b}_{2}=12 N+2 .
$$

Thus we have

$$
\begin{aligned}
& E_{1}^{0, t}=K H^{t+4 N+1, j+12 N+2}(U), \\
& E_{1}^{1, t}=K H^{t+4 N+2, j+12 N+3}(U \sqcup U), \\
& E_{1}^{2, t}=K H^{t+2, j+2}(T(3,3 N)) .
\end{aligned}
$$

For $s=0$ we must have $j$ in the range $-12 N-3 \leq j \leq-12 N-1$, for $s=1$ in the range $-12 N-5 \leq j \leq-12 N-1$ and for $s=2$ in the range $-12 N-3 \leq j \leq-6 N+1$. For $j>-12 N-1$, as in the previous case, we instantly see that the result is as claimed. For the three remaining $j$-values we have $E_{1}$-pages as given in Figure 10.
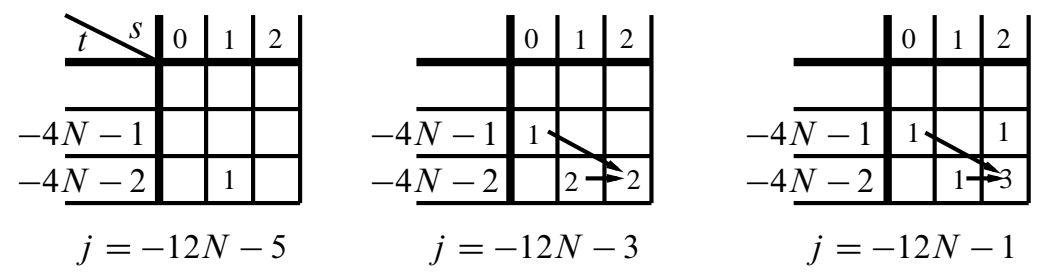

Figure 10: $E_{1}$-pages for Claim 2

For $j=-12 N-5$ there are no differentials for dimensional reasons, but for $j=$ $-12 N-3$ and $j=-12 N-1$ there are possible differentials. The situation is presented in Figure 11, where, as above, possible generators are circled.

Consider the two possible generators in bidegree $(-4 N,-12 N-3)$. Generators in this bidegree would appear in the $E_{\infty}$-page of Lee's spectral sequence. However, $T(3,3 N+1)$ is a knot so the $E_{\infty}$-page has only two generators and these lie on the line $s+t=0$.

Now look at the $E_{1}$-page for $j=-12 N-3$. We have just argued that the two generators on the line $s+t=-4 N$ must be killed. There are two possible ways this might happen, but either way one is left with one generator on the line $s+t=-4 N-1$ and this must survive to $E_{\infty}$.

A similar argument holds for the two possible generators in bidegree $(-4 N-1$, $-12 N-1)$ and one is left with one generator in bidegree $(-4 N,-12 N-1)$. 


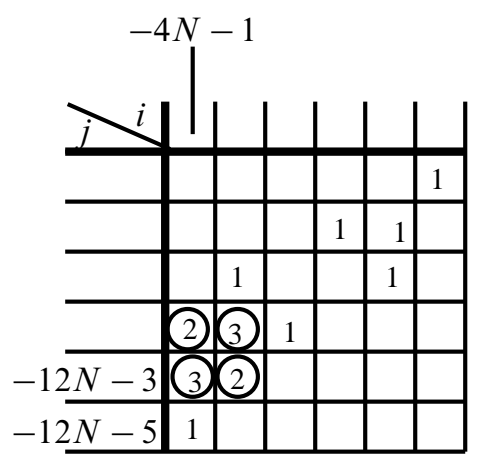

Figure 11: Generators and possible generators for $T(3,3 N+1)$

Proof of Claim 3 This is very similar to the previous arguments so we present this case only briefly. We follow the same orientation convention as above for the diagrams. We have

$$
\begin{aligned}
& E_{1}^{0, t}=K H^{t+4 N+3, j+12 N+8}(U), \\
& E_{1}^{1, t}=K H^{t+4 N+3, j+12 N+6}(U), \\
& E_{1}^{2, t}=K H^{t+2, j+2}(T(3,3 N+1)) .
\end{aligned}
$$

For $j>-12 N-5$ there is nothing to do and for the remaining $j$-values of interest we have $E_{1}$-pages as given in Figure 12 leading to the generators and possible generators presented in Figure 13.
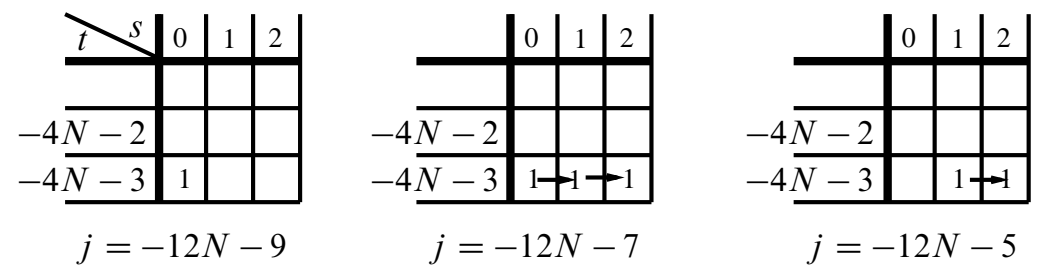

Figure 12: $E_{1}$-pages for Claim 3

As above it is easy to see that the possible generator in bidegree $(-4 N-3,-12 N-7)$ cannot survive to $E_{\infty}$ in Lee's spectral sequence so must be killed. There is only one possibility, leaving one generator in homological degree $-4 N-1$. When $j=-12 N-5$ the two generators both survive because they are needed in Lee's spectral sequence to kill the generators in bidegree $(-4 N-3,-12 N-9)$ and $(-4 N,-12 N-1)$. 


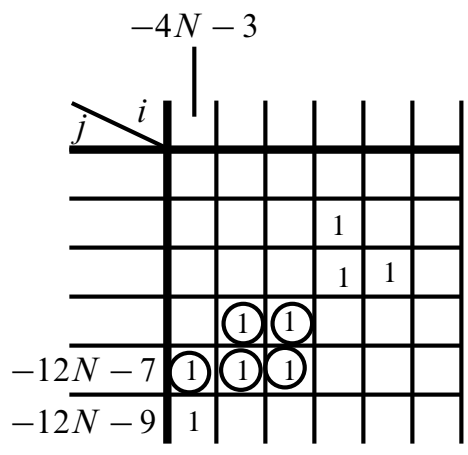

Figure 13: Generators and possible generators for $T(3,3 N+2)$

Finally we note that the inductive process of the above three claims has a beginning because the Khovanov homology of $T(3,2)$ is easily calculated (even by hand), and $T(3,3), T(3,4)$ and $T(3,5)$ can also be computed (by computer or using the spectral sequence - the computations are similar, though not identical, to those above). These cases are seen to have the required form.

Corollary 3.2 The rational Khovanov homology of the torus links $T(3,3 N)$, $T(3,3 N+1)$ and $T(3,3 N+2)$ occupy exactly $N+2$ diagonals.

Since Lee theory over $\mathbb{F}_{p}$ for $p \neq 2$ behaves identically to Lee theory over $\mathbb{Q}$ (in particular Proposition 2.3 holds) the above theorem also calculates $K H^{* * *}\left(D ; \mathbb{F}_{p}\right)$.

Corollary 3.3 The integral Khovanov homology of $T(3, q)$ has no $p$-torsion for $p$ odd.

Proof Since $\operatorname{dim} K H^{i, j}\left(T(3, q) ; \mathbb{F}_{p}\right)=\operatorname{dim} K H^{i, j}(T(3, q) ; \mathbb{Q})$ the result follows immediately from the universal coefficient theorem.

The Khovanov homology of positive crossing $(3, q)$-torus links can be computed from the above by recalling that the rational Khovanov homology of the mirror image $L^{!}$of a link $L$ can be computed as $K H^{i, j}\left(L^{!}\right)=K H^{-i,-j}(L)$.

Remark The result presented in Theorem 3.1 has been independently proven recently by M Stošić in [7], by using the only the skein long exact sequence and a number of intermediate diagrams and complexes.

Acknowledgements Thanks to M Mackaay and J Rasmussen for comments on an early version. 


\section{References}

[1] D Bar-Natan, On Khovanov's categorification of the Jones polynomial, Algebr. Geom. Topol. 2 (2002) 337-370 MR1917056

[2] D Bar-Natan, Khovanov's homology for tangles and cobordisms, Geom. Topol. 9 (2005) 1443-1499 MR2174270

[3] D Bar-Natan, Fast Khovanov homology computations, J. Knot Theory Ramifications 16 (2007) 243-255 MR2320156

[4] M Khovanov, A categorification of the Jones polynomial, Duke Math. J. 101 (2000) 359-426 MR1740682

[5] E S Lee, An endomorphism of the Khovanov invariant, Adv. Math. 197 (2005) 554-586 MR2173845

[6] J Rasmussen, Khovanov homology and the slice genus arXiv:math.GT/0402131

[7] M Stošić, Homology of torus links arXiv:math.GT/0606656

[8] P R Turner, Calculating Bar-Natan's characteristic two Khovanov homology, J. Knot Theory Ramifications 15 (2006) 1335-1356 MR2286127

[9] O Viro, Remarks on the definition of the Khovanov homology arXiv: math.GT/0202199

Maxwell Institute for Mathematical Sciences, Heriot-Watt University

Edinburgh EH14 4AS, Scotland, United Kingdom

paul@ma.hw.ac.uk

Received: 6 November 2007

Algebraic $\&$ Geometric Topology, Volume 8 (2008) 